\title{
Reliability Analysis of Numerical Control Lathe Based On The Field Data
}

\author{
Fei Chen ${ }^{1, a^{*}}$, Xiaojuan Chen ${ }^{2, b}$ and Qunya Xie ${ }^{3, c}$, Binbin Xu ${ }^{4, d}$ \\ ${ }^{1,2,3,4}$ College of Mechanical Science and Engineering, Jilin University, Changchun 130025, China \\ ${ }^{1,2,3,4}$ Key Laboratory of CNC Equipment Reliability Technique of Machinery Industry, \\ Jilin University, Changchun 130025, China \\ achenfeicn@jlu.edu.cn, ${ }^{b}$ chenxj14@mails.jlu.edu.cn, \\ ${ }^{c}$ xieqy14@mails.jlu.edu.cn, ${ }^{d}$ xubinbinjlu@foxmail.com
}

\begin{abstract}
Keywords: numerical control lathe, failure data, reliability analysis, Anderson -Darling(AD) test,repairable system.

Abstract. Numerical control (NC) lathe plays an important role in the production of mechanical parts. Because of its complicated structures and frequent operation, there exists lots of failures. When the failure happens, the NC lathe stops working, then directly lower the productive efficiency. In order to help the users and manufactures to take some maintenance measures to improve the reliability of the NC lathe, in this study, the operating data and failure data of ten NC lathe from 2009 to 2014 were recorded, the statistical description of the failure data were presented, the best fit of failure data were carried out by the Anderson-Darling (AD) test, and the dynamic reliability value were computed, finally, some maintenance measures can be suggested.
\end{abstract}

\section{Introduction}

Numerical control (NC) lathe is the main type of the numerical control machine tool, it is a kind of automatic machine tools with high precision, high speed, high efficiency and extensive processing performance. However, the failures happened during the operation of the NC lathe effect the economic benefits, $\mathrm{NC}$ lathe is repairable system, it can be repaired when faults, because of the complex structure of the NC lathe, its restoration behaviors are difficult and expensive[1]. In recent years, the reliability of numerical control machine tools are increasingly valued.

At present, there exists plentiful literature on the reliability analysis of the products. E.zio [2] described the old problems and new challenges of the reliability engineering. Tsarouhas, et al. [3] pointed out that the equipment's failures cause an additional production gap in the line. In his another literature [4], which analyzed the dialysis system, the reliability characteristics of the subsystem was computed. Regattieri et al. [5] introduced a framework defining a general approach for failure process modeling, it also emphasized the importance of the censored data. To the machine tools, Jia et al. [6] highlights the basis of the data collection on the reliability analysis. Wang et al .[7] analyzed the failure position and subsystem failure mode and cause to indicate the weak subsystem of CNC lathe, Mazzola et al. [8] described the failure distribution of the repairable groups through a non-homogeneous Poisson process. Seungwoo et al.[9] adopted the failure database and weak point analysis to calculate the failure rate, the mean time between failures(MTBF) and other factors about the tool post. Karyagina et al.[10] analyzed the maintenance records about $7 \mathrm{CNC}$ machines and 2 machining centers to find out that mechanical and electrical subsystems each caused the biggest number of breakdowns. Chen et al .[11] presented the descriptive statistics of the failure data and develops the failure trend using power-law process for machining center. In another study, Dr.A.B et al. [12] analyzed the failures of lathe, drilling and press brake machine tools, it concluded that the most critical subsystem of all three machine tools was electrical system. Moreover, Mitchell et al. [13] described a simple technique for estimating the MTBF of a system that has periodic maintenance at regular intervals. While the study of the reliability analysis about the $\mathrm{CNC}$ lathe should go further.

In this paper, the statistical description of the subsystems of the NC lathe was provided, the optimal distribution model of the field data for the subsystem was carried out and the corresponding parameters 
were computed, then the dynamic reliability values were obtained.These can be useful tools to estimate the reliability of the NC lathe, and it contributes to do some preventative maintenance of the NC lathe.

\section{Simple description about the NC lathe and the data collection}

The NC lathe, with extensive processing performance, it can process straight cylindrical, slash cylindrical, circular arc and a variety of complex artifacts, such as thread, trough and worm, it also has the function of line interpolation and circular interpolation.

In this paper, we study the machine tool produced by a certain company, the machine belongs to half closed loop control system of full-function $\mathrm{CNC}$ lathe. We recorded the operating data and failure data about ten NC lathe. The data included failure phenomenon, failure locations, failure reason, failure time, fault repair behavior and fault repair time.

The NC lathe consists of basic components (BC), spindle unit (SU), feeding system (FS), turret (T), pneumatic system (PS), lubrication system (LS), cooling system (CS), hydraulic system (HS), chip conveyor (CC), guard system (GS), numerical control system (NCS), electrical system (ES), detection system (DS) and machine tool accessories (MTA). These fourteen subsystems are series systems, whichever subsystem faults will result in the downtime of the whole machine.

The system reliability index includes: time between failures(TBF), reliability and failure rate. TBF refers to the end time of the previous failure to the start time of the next failure. The longer the TBF is, the greater reliability performance the product represents. Reliability means within the required time and under the specified conditions, the probability the product operating normally. Failure rate refers to the probability of failure happens within certain time period, and before this period of time no failure happened.

\section{Analysis about the failure data of the NC lathe}

In order to find the weak subsystems of the NC lathe, we calculated the number of failures about each subsystem happened from 2009 to 2014, and described the number of failures, failure frequency and the corresponding subsystem in the Pareto chart. As is shown in Fig.1, the higher failure frequency about the subsystems in sequence were: HS, ES, T, FS, MTA, GS, NCS and CS. The number of failures about these subsystems accounted for $95.28 \%$ of the whole system. Oppositely, CC, SU, DS, LS, PS and BC happened few failures or even didn't fault during the observation time, the number of failures about these subsystems accounted for only $4.72 \%$ of the whole system. Therefore, these six subsystems just made a little contribution to the reliability of the NC lathe, we don't study them in the following research, and subsystems mentioned following will not contain them.

As is indicated in Table1, descriptive statistics are applied to describe the basic features for the $\mathrm{TBF}(\mathrm{h})$, the TBF are calculated according to each subsystem, and then the mean, the maximum, the minimum, the range (the maximum minus the minimum), standard deviation (SD), coefficient of variation $(\mathrm{CV})$, kurtosis and skewness of the failure data for the subsystems are figured from the TBF . $\mathrm{SD}$ refers to the arithmetic square root of the variance, the $\mathrm{CV}$ is defined as the ratio of the SD over the mean of the sample, the value of the CV can reflect the degree of dispersion over the data . The larger the $\mathrm{CV}$ is, the greater the dispersion over the data is . The kurtosis reflects the degree of the flat about the frequency distribution curve, and the skewness represents the symmetry of a set of data, if the value of skewness is less than zero, it means that the average is on the left side of the modal, otherwise, the average is on the right side of the modal.

From Table1, we can obtain the following several conclusions: the TBF ranges from $7 \mathrm{~h}$ (HS) to $23158 \mathrm{~h}$ (HS), moreover, the value of the CV about HS is 1.739398, it is the largest over the eight subsystems, it indicates that the HS possesses the greatest randomness. For these subsystems, their value of $\mathrm{CV}$ is more than 1 , it also implies that all the subsystems have large randomness. Meanwhile, the value of skewness is more than zero, it manifests the average is on the right side of the modal, it is a kind of right distribution, also called positively biased. 


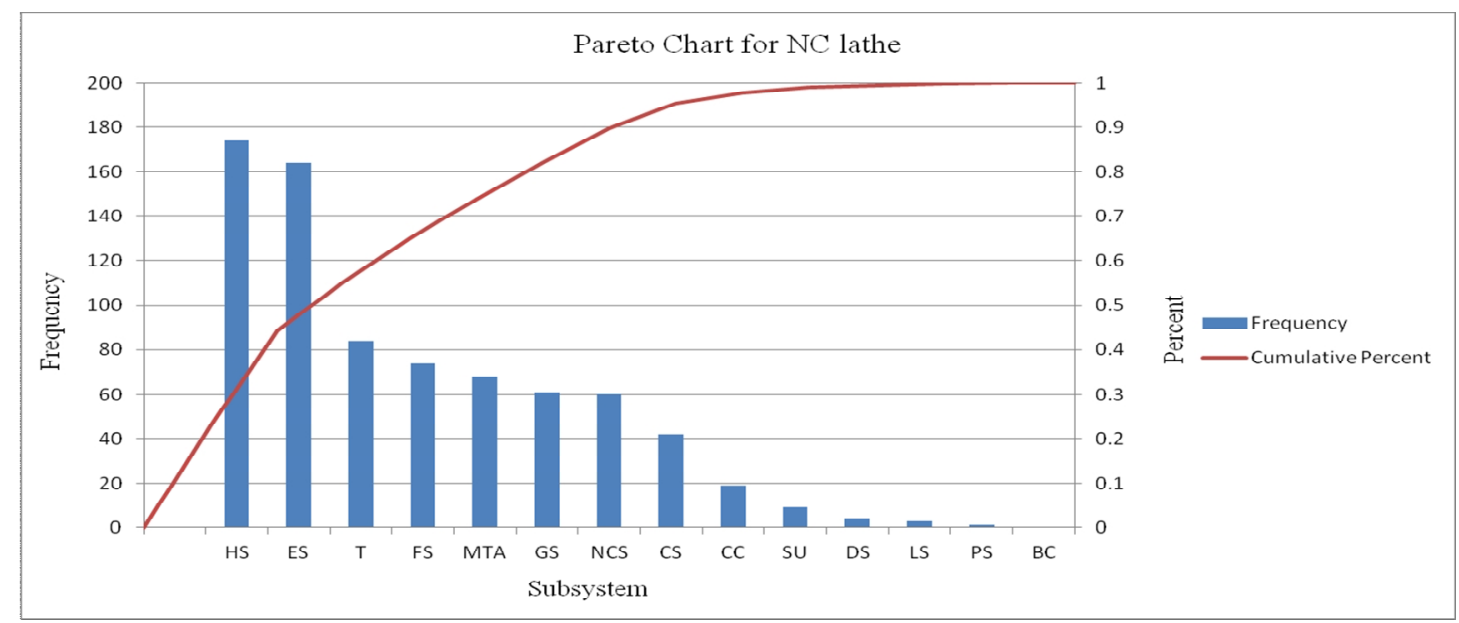

Fig.1 The Pareto chart about the failure location of the NC lathe

Table1 The statistical description of the TBF for each subsystem

\begin{tabular}{cccccccccc}
\hline Subsystem & N & Mean & maximum & Minimum & Range & SD & CV & Kurtosis & Skewness \\
\hline HS & 174 & 1678 & 23158 & 7 & 23151 & 2919 & 1.73957 & 3.9962 & 24.143 \\
ES & 164 & 1785 & 17451 & 14 & 17437 & 2326 & 1.30308 & 3.5137 & 21.035 \\
T & 84 & 2566 & 12500 & 23 & 12477 & 2733 & 1.06508 & 1.6319 & 5.33 \\
FS & 74 & 2809 & 13615 & 16 & 13599 & 3274 & 1.16557 & 1.5856 & 4.9174 \\
MTA & 68 & 2825 & 10995 & 59 & 10936 & 2980 & 1.05486 & 1.4846 & 4.1672 \\
GS & 61 & 2235 & 12182 & 192 & 11990 & 2832 & 1.26711 & 2.0118 & 6.3291 \\
NCS & 60 & 4193 & 20943 & 19 & 20924 & 4608 & 1.09897 & 1.5701 & 5.5178 \\
CS & 42 & 4066 & 16882 & 270 & 16612 & 4500 & 1.10673 & 1.4718 & 4.1263 \\
\hline
\end{tabular}

\section{Modeling of the failure data}

In this chapter, we adopt a goodness-of -fit test of Anderson-Darling (AD) to select the distribution of $\mathrm{TBF}$ for the eight subsystems. Because the AD test can avoid the grouping problem of the chi-square test, and when the sample size is small, the $\mathrm{AD}$ test can acquire a better recognition performance than $\mathrm{k}-\mathrm{s}$ test. In a general way, the distribution of $\mathrm{TBF}$ for $\mathrm{NC}$ lathe usually considers as exponential distribution, normal distribution, lognormal distribution and weibull distribution. In matlab programming method, we adopt $\mathrm{AD}$ to test the failure data of the subsystems. Assume the significance level value is 0.05 , and we can attain the value of $h, p$ and adstat. $h$ is the hypothesis test result, which retuned as a logical value. $p$-value of the $A D$ test,returned as a sclar value in the range $[0,1], p$ is the probability of observing a test statistic as extrene as, or more extreme than, the observed value under the null hypothesis. Adstat defined as the test statistic for the AD test, returned as a scalar value, the smaller the adtest is, the better the data obeys the distribution. When the value of $\mathrm{p}$ is less than 0.05 , it indicates that the data doesn't obey the distribution, and the value of $h$ equals to 1 ; when the value of $p$ is more than 0.05 , it represents that the data obeys the distribution, and the value of h equals to 0 ; when the value of $h$ equals to 0 , the larger the $p$ is, the better the failure data obeys the distribution, mean while, the smaller the value of the adstat is , the better the data obeys the distribution. Table 2 shows the results of the $\mathrm{AD}$ test, except the MTA, the other seven subsystems attained their best fit satisfied the largest value of $\mathrm{p}$ and the smallest value of adstat simultaneously. For the MTA, when it obeys exponential distribution, it has the largest value of $\mathrm{p}$, but when it obeys the lognormal distribution, it has the smallest value of adstat. Here, we choose the exponential distribution as the best fit of the MTA. It also can be concluded that the distribution of the TBF for the eight subsystems doesn't obey the normal distribution, they tend to obey lognormal distribution and weibull distribution, while the TBF of 
MTA obeys the exponential distribution. The best distribution of the TBF for each subsystem can be obtained from the Table 2. Such as, for the FS, the best fit is weibull distribution.

Table 2 The results of AD-test for the subsystems

\begin{tabular}{|c|c|c|c|c|c|c|c|c|c|c|c|c|c|}
\hline & \multicolumn{3}{|c|}{ Exponential } & \multicolumn{3}{|c|}{ Normal } & \multicolumn{3}{|c|}{ Lognormal } & \multicolumn{3}{|c|}{ Weibull } & \multirow[t]{2}{*}{ Best-fit } \\
\hline & $\mathrm{h}$ & $\mathrm{p}$ & adstat & $\mathrm{h}$ & $\mathrm{p}$ & adstat & $\mathrm{h}$ & $\mathrm{p}$ & adstat & $\mathrm{h}$ & $\mathrm{p}$ & adstat & \\
\hline FS & 1 & 0.0051 & 2.2309 & 1 & 0.0005 & 4.1793 & 0 & 0.0817 & 0.6575 & 0 & 0.5457 & 0.3249 & Weibull \\
\hline $\mathrm{T}$ & 0 & 0.4438 & 0.5346 & 1 & 0.0005 & 4.1791 & 1 & 0.0043 & 1.1685 & 0 & 0.9403 & 0.1760 & Weibull \\
\hline $\mathrm{CS}$ & 0 & 0.4176 & 0.5539 & 1 & 0.0005 & 2.3735 & 0 & 0.8465 & 0.2118 & 0 & 0.3375 & 0.4159 & Lognormal \\
\hline HS & 1 & 0.0005 & 14.3679 & 1 & 0.0005 & 20.6824 & 0 & 0.6139 & 0.2966 & 1 & 0.0113 & 1.0210 & Lognormal \\
\hline GS & 1 & 0.0102 & 1.9377 & 1 & 0.0005 & 5.4349 & 0 & 0.1302 & 0.5752 & 1 & 0.0008 & 1.4520 & Lognormal \\
\hline NCS & 0 & 0.0683 & 1.1934 & 1 & 0.0005 & 2.6594 & 0 & 0.0928 & 0.6329 & 0 & 0.3165 & 0.4280 & Weibull \\
\hline ES & 1 & 0.0029 & 2.4757 & 1 & 0.0005 & 11.0430 & 1 & 0.0094 & 1.0395 & 0 & 0.3278 & 0.4227 & Weibull \\
\hline MTA & 0 & 0.4297 & 0.5462 & 1 & 0.0005 & 4.1115 & 0 & 0.4120 & 0.3728 & 0 & 0.2341 & 0.4819 & Exponential \\
\hline
\end{tabular}

\section{Reliability analysis of the subsystems and the whole machine}

Reliability is the probability that the product works normally under specifed conditions, for a given period of time. Assume the T refers to the TBF, $T \geq 0$, the reliability function $R(t)$ can be defined as

$$
R(t)=\operatorname{Pr}\{T \geq t\}
$$

As described earlier, for the NC lathe, its reliability depends on the eight subsystems, moreover, they are series systems, whichever subsystem faults, the whole machine will stop operation.

The reliability diagram of the NC lathe is shown in Fig.2.

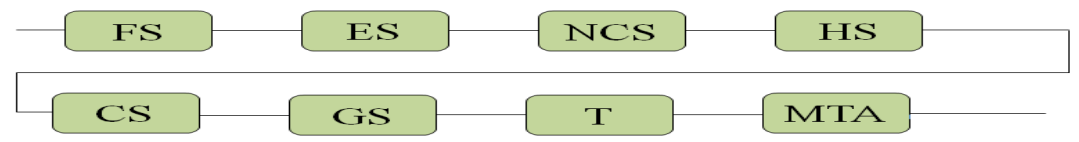

Fig.2 The reliability diagram of the NC lathe

The reliability of the whole machine can be calculated as,

$$
R=R_{F S} R_{E S} R_{N C S} R_{H S} R_{C S} R_{G S} R_{T} R_{M T A}
$$

Use the maximum likelihood estimation method to estimate the parameter values of the distribution, the results are shown in Table 3.

\begin{tabular}{|c|c|c|c|c|c|c|c|}
\hline \multicolumn{2}{|c|}{ FS } & \multicolumn{2}{|c|}{$\mathrm{T}$} & \multicolumn{2}{|c|}{$\mathrm{CS}$} & \multicolumn{2}{|c|}{ HS } \\
\hline \multicolumn{2}{|c|}{ weibull } & \multicolumn{2}{|c|}{ weibull } & \multicolumn{2}{|c|}{ lognormal } & \multicolumn{2}{|c|}{ lognormal } \\
\hline shape & scale & shape & scale & mean & variance & mean & variance \\
\hline 0.79301 & 2465 & 0.90173 & 2444 & 7.7089 & 1.1722 & 6.3741 & 1.5817 \\
\hline \multicolumn{2}{|c|}{ DS } & \multicolumn{2}{|c|}{ NCS } & \multicolumn{2}{|c|}{ ES } & \multicolumn{2}{|c|}{ MTA } \\
\hline \multicolumn{2}{|c|}{ lognormal } & \multicolumn{2}{|c|}{ weibull } & \multicolumn{2}{|c|}{ weibull } & \multirow{2}{*}{\multicolumn{2}{|c|}{$\begin{array}{l}\text { exponential } \\
\text { failure rate }\end{array}$}} \\
\hline mean & variance & shape & scale & shape & scale & & \\
\hline 7.1006 & 1.0878 & 0.85158 & 3864 & 0.84941 & 1631 & 0.0 & 5394 \\
\hline
\end{tabular}

Table 3 The parameter values of the distribution

From the Table 3, we can calculated the reliability of the subsystems and the whole machine in a given time interval. Table 4 shows the reliability values for the eight subsystems and the whole machine. 
$R_{S}$ refers to the reliability of the whole machine.

Table4 The reliability values of the subsystems and the whole machine for different time

\begin{tabular}{cccccccccc}
\hline Time $(\mathrm{h})$ & $R_{F S}$ & $R_{T}$ & $R_{C S}$ & $R_{H S}$ & $R_{D S}$ & $R_{N C S}$ & $R_{E S}$ & $R_{M T A}$ & $R_{S}$ \\
\hline 5 & 0.992706 & 0.996248 & 1 & 0.998703 & 1 & 0.996534 & 0.992698 & 0.998232 & 0.952564 \\
10 & 0.987396 & 0.993001 & 0.999998 & 0.994975 & 0.999995 & 0.993754 & 0.986882 & 0.996467 & 0.913494 \\
30 & 0.970142 & 0.981263 & 0.999881 & 0.969916 & 0.999664 & 0.984159 & 0.966984 & 0.989438 & 0.778976 \\
50 & 0.955565 & 0.970463 & 0.9994 & 0.940217 & 0.998312 & 0.975632 & 0.949507 & 0.982459 & 0.668206 \\
100 & 0.924267 & 0.945525 & 0.995949 & 0.868296 & 0.989105 & 0.956461 & 0.910872 & 0.965225 & 0.461949 \\
150 & 0.89707 & 0.922433 & 0.989329 & 0.805663 & 0.972652 & 0.939062 & 0.876573 & 0.948294 & 0.323462 \\
200 & 0.872444 & 0.900636 & 0.980131 & 0.751793 & 0.951221 & 0.922814 & 0.845184 & 0.931659 & 0.228499 \\
250 & 0.8497 & 0.879871 & 0.968986 & 0.705078 & 0.926705 & 0.907431 & 0.816031 & 0.915317 & 0.162573 \\
300 & 0.828443 & 0.859978 & 0.956419 & 0.664142 & 0.900442 & 0.892746 & 0.78871 & 0.899261 & 0.116393 \\
400 & 0.789436 & 0.822403 & 0.928558 & 0.595576 & 0.846044 & 0.865067 & 0.738557 & 0.867989 & 0.060651 \\
500 & 0.754119 & 0.787332 & 0.898806 & 0.540159 & 0.792315 & 0.839219 & 0.693292 & 0.837805 & 0.032222 \\
600 & 0.72173 & 0.754401 & 0.868481 & 0.494242 & 0.741143 & 0.814872 & 0.652033 & 0.80867 & 0.017411 \\
700 & 0.691766 & 0.723351 & 0.838358 & 0.455454 & 0.693279 & 0.791803 & 0.614167 & 0.780549 & 0.00955 \\
800 & 0.663869 & 0.693988 & 0.808891 & 0.422181 & 0.648922 & 0.769853 & 0.57924 & 0.753405 & 0.005309 \\
1000 & 0.613256 & 0.639722 & 0.75284 & 0.367910 & 0.570356 & 0.728845 & 0.516855 & 0.701917 & 0.0017 \\
1500 & 0.509454 & 0.525235 & 0.63215 & 0.276342 & 0.422517 & 0.639716 & 0.394021 & 0.58807 & 0.000116 \\
\hline
\end{tabular}

As is shown in Table 4, with the increase of the time, the reliability value declined, and the whole machine decreased most greatly. when the time is less than $100 \mathrm{~h}$, the reliability value of the subsystem was around $90 \%$, while the whole machine was less than $50 \%$, when the time increased to $400 \mathrm{~h}$, the reliability value of the whole machine decreased to less than $10 \%$, when the time was more than $1500 \mathrm{~h}$, the reliability value of the whole machine was close to zero. So if we want to improve the reliability of the whole machine, we should focus on the weak subsystems to improve the reliability of the whole machine.

\section{The measures to improve the reliability of the NC lathe}

As is shown in Table1, the HS and ES had the highest failure frequency, their MTBF was the smallest, to improve the reliability of the NC lathe, it can improve the reliability of the HS and ES. About the all failures, the failures of the HS accounted for $22.8 \%$, and the ES accounted for $21.49 \%$. The Table 5 and Table 6 show the main failure phenomenon, failure cause and the corresponding repair measures of the HS and ES respectively.

Table 5 The main failure phenomenon, failure cause and corresponding repair measures of the HS

\begin{tabular}{|c|c|c|c|}
\hline number & Failure phenomenon & Failure causes & Maintenance measures \\
\hline 1 & Tool doesn't turn or chuck doesn't move & Solenoid valve gets stuck & $\begin{array}{l}\text { Clean or adjust the Solenoid } \\
\text { valve }\end{array}$ \\
\hline 2 & Oil leaks from the hydraulic pump & Oil seal damages & Replace the oil seal \\
\hline 3 & Abnormal sound from the hydraulic pump & Bearing damages & Replace the bearing \\
\hline 4 & Chuck failures & Rotary cylinder disengaged & reinstall \\
\hline 5 & $\begin{array}{c}\text { Oil leaks from the hydraulic pressure } \\
\text { station }\end{array}$ & Oil tube interface damages & Replace the interface \\
\hline 6 & Hydraulic pressure is instable & Pump head damages & Replace the pump head \\
\hline 7 & Hydraulic system failures & Oil leaks or less oil & Tighten the oil tube and add oil \\
\hline 8 & Hydraulic system alarms & $\begin{array}{l}\text { the motor bearing of the oil } \\
\text { pump damages }\end{array}$ & Replace the bearing \\
\hline
\end{tabular}

From Table 5, the HS had many failure phenomenon and failure causes, and from their repair measures, some conclusions can be made, the failure phenomenon number 2, 3, 5, 6 and 7 replaced the failure parts to restore the failure; oil leaks was a common failure phenomenon. Therefore, to improve the reliability of the HS, the following measures can be taken: (1) improve the quality of the key components of the HS, such as, tubing, pump head, oil seal and so on; (2) solenoid valve should be cleaned at regular intervals; (3) pay attention to the hydraulic pressure. As is shown in Table 6, the failures of the ES mainly came from the lines and the relay, the periodic inspection and repair for the 
lines are necessary, especially for the $24 \mathrm{v}$ power supply, and it is also needed to look out whether the input voltage is high and the connection between the lines is correct. Clean the scrap iron inside the machine in time, to avoid iron block cause the malfunction of the machine.

Table 6 The main failure phenomenon, failure cause and corresponding repair measures of the ES

\begin{tabular}{cccc}
\hline number & Failure phenomenon & Failure causes & Maintenance measures \\
\hline 1 & Chuck doesn't move & Relay damages & Replace the relay \\
2 & The machine can't boot & The $24 \mathrm{v}$ power supply burns & Replace the power supply \\
3 & The spindle doesn't turn & Short circuit of power lines & Line hunting \\
4 & 607、613 and 9004 alarm & Drive power supply has much iron & Clean the iron filings \\
5 & Machine tool line catches fire & The line to ground fault & Restitute the line \\
6 & 2043 alarms & Abnormal pressure switch induction & $\begin{array}{c}\text { Pressure switch short } \\
\text { connected } \\
7\end{array}$ \\
2049 alarms & Line failures & Repair the line \\
\hline
\end{tabular}

\section{Conclusions}

Improve the reliability of the NC lathe has significant importance for the machine industry. The main conclusions about this study are as follows:

(1) From the subsystems perspective, the TBF (HS) ranged from $7 \mathrm{~h}$ to $23158 \mathrm{~h}$ (HS), it indicated that the TBF of the HS had the largest randomness, compared with other subsystems, it also had the largest range;

(2) The HS had the smallest mean TBF, followed by the ES, the NCS had the largest mean TBF, which showed that the HS and the ES had high failure frequency, while the NCS had low failure frequency;

(3) The reliability value of subsystems and the whole machine at different TBF were computed, which can provide the power basis for the user and the machine tool manufacturing enterprise to make some improvements;

(4) List the main failure phenomenon, failure cause and the corresponding repair measures of the HS and ES, and give some constructive suggestions to improve the reliability of the HS and ES.

Finally, to improve the reliability of the NC lathe, the manufacturers should focus on improving the quality of the HS and ES firstly, and followed by T, FS, MTA, GS, NCS and CS . CC, SU ,DS, PS and $\mathrm{BC}$ happened few failures, or even didn't failure in the observation time, we can allocate high reliability value to these six subsystems.

\section{Acknowledgement}

This work is supported by the Specialized Research Fund of the One Thousand sets of Domestic CNC lathe Reliability Engineering ( Grant No. 2013ZX04011-011) and the project of Jilin province science and technology development plan of the Machining Center Reliability Modeling of Cutting Load (Grant No. 3D513S292414).

\section{References}

[1] Keller A Z, Kamath A R R, Perera U D. Reliability analysis of CNC machine tools[J]. Reliability Engineering, 1982, 3(6):449-473.

[2] Zio E. Reliability engineering: Old problems and new challenges[J]. Reliability Engineering \& System Safety, 2009, 94(2):125-141.

[3] Tsarouhas P H, Varzakas T H, Arvanitoyannis I S. Reliability and maintainability analysis of strudel production line with experimental data - A case study[J]. Journal of Food Engineering, 2009, 91(2):250-259. 
[4] Tsarouhas, P H. Reliability Analysis into Hospital Dialysis System: A Case Study[J]. Quality \& Reliability Engineering International, 2013, 29(8):1235-1243.

[5] Regattieri A, Manzini R, Battini D. Estimating reliability characteristics in the presence of censored data: A case study in a light commercial vehicle manufacturing system[J]. Reliability Engineering \& System Safety, 2010, 95(10):1093-1102.

[6] Jia Y, Wang M, Jia Z. Probability distribution of machining center failures[J]. Reliability Engineering \& System Safety, 1995, 50(1):121-125.

[7] Wang Y, Jia Y, Yu J, et al. Failure probabilistic model of CNC lathes[J]. Reliability Engineering \& System Safety, 1999, 65(3):307-314.

[8] Mazzola M, Merlo A, Aggogeri F, et al. Analysis of Machine Tool Failures Using Advanced Reliability Models for Complex Repairable Systems[J]. American Society of Mechanical Engineers, 2008:163-171.

[9] Seungwoo Lee, Seungwoo Han , Husang Lee. Assessment of the Tool Post Reliability of a High-Stiffness Turning Machine[J].Mechanical Science and Technology,2007:1244-1252.

[10] Karyagina M, Wong W, Vlacic L. Reliability aspect of CNC machines-are we ready for integration?[C]. Emerging Technologies and Factory Automation, 1995.

[11] Chen, Chuaihai et al. Reliability analysis of machining center based on the field data [J]. Eksploatacja I Niezawodnosc-Maintenance and Reliability, 2013; (2): 147

[12] Dr.A.B.Andhare, C.Kant Tiger, Sarfraj Ahmed . Failure Analysis of Machine Tools using GTMA and MADM method[J]. International Journal of Engineering Research \& Technology,2012.

[13] Mondro M J. Approximation of mean time between failure when a system has periodic maintenance[J]. Reliability IEEE Transactions on, 2002, 51(2):166 - 167. 